\title{
Research Article \\ Simvastatin-Loaded Nanomicelles Enhance the Osteogenic Effect of Simvastatin
}

\author{
Xianling Feng $\mathbb{D},{ }^{1}$ Xinxin Yue $\mathbb{D},{ }^{2}$ and Mao Niu $\mathbb{D}^{3}$ \\ ${ }^{1}$ School of Medicine, Shenzhen University, Shenzhen 518060, China \\ ${ }^{2}$ Department of Dental Implantology, Beijing Stomatological Hospital, School of Stomatology, Capital Medical University, \\ Beijing 100050, China \\ ${ }^{3}$ School of Medical Technology and Nursing, Shenzhen Polytechnic, Shenzhen 518055, China
}

Correspondence should be addressed to Mao Niu; niu1037@szpt.edu.cn

Received 26 June 2020; Revised 30 October 2020; Accepted 5 November 2020; Published 19 November 2020

Academic Editor: Xiaoming Li

Copyright (c) 2020 Xianling Feng et al. This is an open access article distributed under the Creative Commons Attribution License, which permits unrestricted use, distribution, and reproduction in any medium, provided the original work is properly cited.

Objectives. The present study intended to further verify that simvastatin-loaded nanomicelles (SVNs) enhanced the role of simvastatin (SV) in promoting osteoblast differentiation in vitro and to evaluate the effect of SVNs on bone defect repair in vivo. Methods. SVNs were synthesized by dialysis. MG63 cells were subjected to intervention with $0.25 \mu \mathrm{mol} / 1$ of SVNs and SV. A 3(4,5-dimethylthiazol-2-yl)-5-(3-carboxymethoxyphenyl)-2-(4-sulfophenyl)-2H-tetrazolium (MTS) assay kit and flow cytometry were used to determine cell proliferation activity, cell cycle distribution, and apoptosis. The osteoblastic differentiation of MG 63 cells was evaluated by measuring alkaline phosphatase (ALP) activity, ALP staining, and the expression levels of the osterix (Osx) and osteocalcin (OC) proteins. In addition, $0.5 \mathrm{mg}$ of SVNs or SV was applied to the skull defect area of rabbits. Micro$\mathrm{CT}$, hematoxylin and eosin (HE) staining, and Masson's trichrome staining were used for qualitative and quantitative evaluation of new bone in three dimensions and two dimensions. Results. The SVNs had a mean diameter of $38.97 \mathrm{~nm}$. The encapsulation and drug-loading efficiencies were $54.57 \pm 3.15 \%$ and $10.91 \pm 0.63 \%$, respectively. In vitro, SVNs and SV can inhibit the proliferation activity and promote osteogenic differentiation of MG63 cells by arresting MG63 cells at the G0/G1 phase without increasing the apoptosis rate. In vivo quantitative results showed that the bone mineral density (BMD), bone volume (BV)/total volume (TV) ratio, and trabecular number (Tb.N) in the gelatin sponge with SVNs (SVNs-GS) group and gelatin sponge with SV (SV-GS) group were 362.1\%, 292.0\%; 181.3\%, 158.0\%; and 215.2\%, 181.8\% of those in the blank control (BC) group, respectively. Histological results identified the new bone tissue in each group as irregular fibrous bone, and the arrangement of trabecular bone was disordered. There were significantly more osteoblasts and new capillaries around the trabecular bone in the SVNs-GS group and SV-GS group than in both the BC and drug-free nanomicelle (DFNs) groups. Both in vitro and in vivo, SVNs exhibited greater osteogenic efficacy than SV. Conclusion. SVNs significantly improved the osteogenic efficacy of SV.

\section{Introduction}

Trauma, infection, and tumors can all cause bone defects. Due to mineral deposits and an insufficient blood supply in bone defect areas, the rate of new bone formation is very slow, and large bone defects cannot self-heal $[1,2]$. Methods such as bone grafting, bone tissue engineering, and membrane-guided bone regeneration are commonly used to promote bone defect repair, and the materials used include autogenic bone, allogenic bone, synthetic bone replacement materials, stem cells, and exogenous growth factors [3, 4].
Among these materials, autogenic bone is the gold standard of bone repair materials due to its superior osteoconduction, osteoinduction, and osteogenesis [5]. However, autogenic bone grafting is associated with considerable trauma and increased patient suffering, which substantially limits its clinical application [6]. Allogenic bone, which is mainly derived from human cadavers derived, has been applied in clinical practice and has yielded good results [7]. However, the potential risks of spreading viruses and immunological rejection limit the application of allogenic bone in clinical practice [8]. Synthetic biodegradable bone replacement materials 
overcome the limitations of the above two materials but have the disadvantages of fast degradation, low strength, and localized inflammation caused by degraded products [9, 10]. The addition of exogenous osteogenic growth factors (such as bone morphogenetic protein 2 (BMP-2)) to the above materials can significantly improve the osteoinductive ability and promote the proliferation and differentiation of osteoblasts [11-14]. However, exogenous osteogenic growth factors are expensive, have a short half-life, are difficult to store, and have low cell-targeting properties [15]. Therefore, some scholars have attempted to identify specific drugs to accelerate the formation of new bone in the bone defect area by regulating endogenous osteogenic growth factors.

Simvastatin (SV), which is commonly used in clinical practice, is inexpensive, safe, and reliable. SV can specifically bind to HMG-CoA reductase, a key enzyme in cholesterol synthesis, to inhibit cholesterol synthesis and prevent cardiovascular diseases [16]. In 1999, Mundy et al. first reported that subcutaneous injection of SV and lovastatin could significantly promote the formation of new bone in a mouse cranial bone defect area [17]. Studies have shown that SV can promote osteogenesis mainly through the induction of BMP-2 and VEGF gene expression, thereby promoting angiogenesis and the differentiation of osteoblasts $[17,18]$. In addition, SV inhibits the RANKL expression to suppress the differentiation of osteoclasts and promote osteogenesis $[19,20]$. The osteogenesis-promoting effect of SV is closely associated with the regulatory functions of osteogenesisrelated signaling pathways, such as the Smad pathway, the Erk1/2 pathway, and the Pi3k/Akt pathway [21-26].

$\mathrm{SV}$ is liposoluble and poorly soluble in water. When used systemically, most drugs are metabolized by the liver. Only small amounts of drugs can reach bone defect areas. An extremely low available drug concentration seriously impacts the promoting effect of SV [27]. Increasing the dose of SV for systemic administration can effectively increase the drug concentration in the bone defect area, but high doses can also increase the systemic side effects of SV, such as liver and kidney damage and rhabdomyolysis [28, 29]. Therefore, the extremely low bioavailability in bone defect areas and the potential side effects of SV are urgent problems requiring resolution.

Nano-based drug carriers include nanoparticles, nanoliposomes, solid lipid nanoparticles, magnetic nanoparticles, and polymeric nanomicelles. Among these carriers, polymeric nanomicelles, which were first proposed by Ringsdorf et al. to be used as a drug carrier, have become a research focus of drug delivery systems. Amphiphilic polymeric nanomicelles are self-assembled nanostructures formed by hydrophilic shells and a hydrophobic core. Polymeric nanomicelles are mostly spherical in shape and are mainly used for encapsulation and delivery of lipophilic small-molecule drugs [30, 31]. The particle sizes of polymeric nanomicelles are relatively small, mostly ranging between 10 and $80 \mathrm{~nm}$ [32]. Currently, polyethylene glycol (PEG), polyethylene oxide (PEO), and chitosan (CTS) are commonly used in the synthesis of the hydrophilic segments of polymeric nanomicelles [33, 34]. PEG has good water solubility and a large exclusion volume, which can effectively prevent aggregation of micelles.
PEG can also reduce the capture of the reticuloendothelial system (RES) and extend the half-life of polymeric nanomicelles [33, 34]. The hydrophobic segments of polymeric nanomicelles are usually synthesized using polylactic acid (PLA) and polycaprolactone (PCL), which mainly serve the functions of encapsulating hydrophobic drugs [30, 34]. Polymeric nanomicelles prepared with these materials have excellent biocompatibility and biodegradability [35]. Polymeric nanomicelles can be excreted through the kidneys and will not accumulate in the body. Therefore, polymer micelles have great application prospects as drug carriers.

We hypothesized that the encapsulation of SV using polymer nanomicelles can significantly improve the in vitro and in vivo osteogenic effect of SV. In previous studies, we applied methoxy-PEG (mPEG-) PLA to encapsulate SV, successfully synthesized simvastatin-loaded nanomicelles (SVNs) by dialysis, and preliminarily verified that an appropriate concentration of SVNs can significantly improve the osteoblast differentiation-promoting effect of SV in vitro [24]. In the present study, we not only further verified the role of SVNs in promoting osteoblast differentiation in vitro but also evaluated the in vivo osteogenesispromoting effects of SVNs.

\section{Materials and Methods}

2.1. Preparation and Characterization of SVNs. Based on previous experience in preparing nanomicelles in our group [24], SVNs (mPEG-PLA-SV) were prepared using a dialysis method. In short, $10 \mathrm{mg}$ of mPEG-PLA $(\mathrm{Mn}=5000-3000)$ (Jinan Daigang Biomaterial Co., Ltd., Shandong, China) and $2 \mathrm{mg}$ of SV (National Institutes for Food and Drug Control, Beijing, China) were dissolved in $2 \mathrm{ml}$ of acetonitrile (Fisher Scientific, Waltham, MA, USA) and slowly added dropwise into $12 \mathrm{ml}$ of purified water. The mixture was stirred for $1 \mathrm{~h}$ at room temperature and then dispersed via ultrasonication for $5 \mathrm{~min}$. Finally, the above solution was dialyzed for $7 \mathrm{~h}$. The drug-free nanomicelles (DFNs) were also prepared in a similar way in the absence of SV.

The particle sizes of SVNs and DFNs were measured using a Zetasizer instrument (Mastersizer 2000, Malvern Instruments Ltd., Malvern, UK). Nanomicelle morphology was observed with a transmission electron microscope (TEM; HT7700, Hitachi Limited, Tokyo, Japan). Highperformance liquid chromatography (HPLC; LC-20AD, Shimazu Corporation, Kyoto, Japan) was used to determine the drug-loading content and drug entrapment efficiency.

2.1.1. In Vitro Simvastatin Release. SVNs were sealed in a dialysis bag and incubated in $4 \%$ HAS solution at $37^{\circ} \mathrm{C}$ and $50 \mathrm{rpm}$. The same amount of free SV was dialyzed under the same conditions as a control. At predetermined time intervals, $12 \mathrm{ml}$ of the samples was withdrawn and replaced with $12 \mathrm{ml}$ of fresh release medium. The SV concentration was analyzed using the HPLC system.

\subsection{Cell Experiment}

2.2.1. Cell Culture and Experimental Group. The human osteoblast-like MG63 cells were purchased from the Cell 

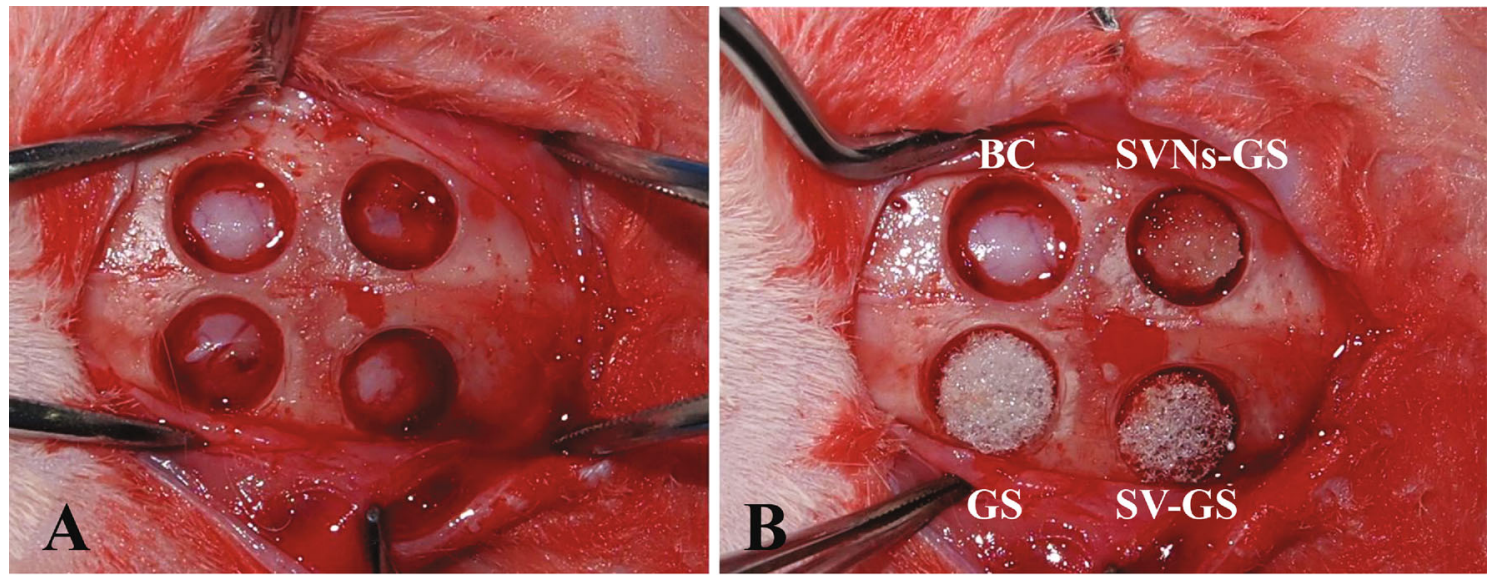

Figure 1: The surgical procedures with rabbit calvaria. (a) A mucoperiosteal flap was raised, and four critical bone defects (6.5 mm in diameter) were created at the top of the skull. (b) The defects were divided into the following four groups: no augmentation (blank control, BC) group, gelatin sponge (GS) group, gelatin sponge with $0.5 \mathrm{mg} \mathrm{SV}$ (SV-GS) group, and gelatin sponge with $5 \mathrm{mg}$ SVNs (0.5 mg SV) (SVNs-GS) group.

Bank of the Chinese Academy of Sciences, Shanghai, People's Republic of China. The MG63 cells were cultured in Dulbecco's modified Eagle's medium (DMEM; Thermo Fisher Scientific, Waltham, MA, USA) with $10 \%$ fetal bovine serum (FBS; Thermo Fisher Scientific, Waltham, MA, USA) and $1 \%$ penicillin-streptomycin (HyClone, Logan, UT, USA) at $37^{\circ} \mathrm{C}$ in a humidified incubator with $5 \% \mathrm{CO}_{2}$. The medium was refreshed every three days.

The experiment was divided into four groups: SVNs group, SV group as the positive control, DFNs group, and blank control (BC) group as the negative control. According to the preliminary results of our group, the SV in the SVNs group and in the SV group was dissolved and diluted to $0.25 \mu \mathrm{mol} / 1$ in the complete medium. Equal concentrations of DFNs were also prepared.

2.2.2. Cell Proliferation. MG63 cells were seeded onto 24 -well plates at $10^{4}$ cells per well and cultured with the drug formulations $(500 \mu \mathrm{l} /$ well $)$ for $72 \mathrm{~h}$. Then, culture medium was replaced with serum-free medium. The plate was incubated for $2 \mathrm{~h}$ at $37^{\circ} \mathrm{C}$ in a humidified atmosphere with $5 \% \mathrm{CO}_{2}$. An MTS assay kit (MTS; Biovision Inc., CA, USA) was used to measure cell proliferation. One hundred microliters of MTS solution was mixed with culture medium per well. One hundred microliters of the mixture was pipetted into each well of a 96-well plate, and absorbance was measured at $30 \mathrm{~min}$ intervals at $490 \mathrm{~nm}$ using a microplate reader (Tecan, Tecan Trading AG, Switzerland).

2.2.3. Cell Cycle and Apoptosis. MG63 cells were seeded in $6 \mathrm{~cm}$ Petri dishes at $1 \times 10^{6} /$ dish. The experiment was terminated at $72 \mathrm{~h}$ after dosing, and the effects of drugs on the cell cycle and apoptosis of MG63 cells were measured by flow cytometry.

(1) Cell Cycle. A cell cycle detection kit (keygen, Keygen biotech, Jiangsu, China) was used to detect the cell cycle. The cells were digested and collected by centrifugation at 1,000 $\times \mathrm{g}$ at $4^{\circ} \mathrm{C}$ for $5 \mathrm{~min}$. With the supernatant removed, the cells were resuspended with $1 \mathrm{ml}$ of precooled Buffer A and collected by centrifugation again. Then, the cells were resuspended with $100 \mu \mathrm{l}$ of precooled Buffer A, slowly dripped into $900 \mu \mathrm{l}$ of precooled $70 \%$ ethanol, and fixed at $-20^{\circ} \mathrm{C}$ for at least $12 \mathrm{~h}$. The cells were collected by centrifugation again, washed with precooled Buffer A to remove ethanol, and resuspended in $500 \mu \mathrm{l}$ of Buffer A; then, RNase A was added, and the solution was further incubated at $37^{\circ} \mathrm{C}$ for $30 \mathrm{~min}$. The samples were stained with propidium iodide (PI) at room temperature for $30 \mathrm{~min}$ in the dark and analyzed by flow cytometry.

(2) Cell Apoptosis. An Annexin V-FITC/PI Apoptosis Detection Kit (BD, Becton, Dickinson and Company, NJ, USA) was used to detect the apoptotic cells. The cells were collected using trypsin without EDTA by centrifugation and resuspended with $300 \mu \mathrm{l} 1 \times$ binding buffer. One hundred microliters of cell suspension was pipetted into culture tubes; then, $5 \mu \mathrm{l}$ of Annexin V-FITC was added to each tube, followed by incubation for $15 \mathrm{~min}$ at room temperature. Five microliters of PI stain was added to the cells for $5 \mathrm{~min}$ at room temperature without light. After the addition of $400 \mu \mathrm{l}$ of $1 \times$ binding buffer to each tube, cell apoptosis was analyzed by flow cytometry.

2.2.4. Alkaline Phosphatase (ALP) Activity. MG63 cells were seeded onto 24-well plates at $10^{4}$ cells/well. After $7 \mathrm{~d}$ of culturing, the culture medium was removed, and $50 \mu \mathrm{l} /$ well of Laemmli sample buffer together with protease inhibitor $(50: 1 \mathrm{v} / \mathrm{v})$ was added to lyse the cells. Then, the samples were centrifuged at $10,000 \mathrm{rpm}$, at $4^{\circ} \mathrm{C}$. Ten microliters of supernatant was pipetted to measure the protein concentration using a bicinchoninic acid protein assay kit (Thermo, Thermo Fisher Scientific, MA, USA). The absorbance of the solution was measured at $562 \mathrm{~nm}$, and the amount of total protein was calculated using a generated calibration curve. In addition, $30 \mu \mathrm{l}$ of supernatant was pipetted to measure the ALP activity, and the absorbance of the solution at $520 \mathrm{~nm}$ was measured. To determine the ALP activity, the amount of 


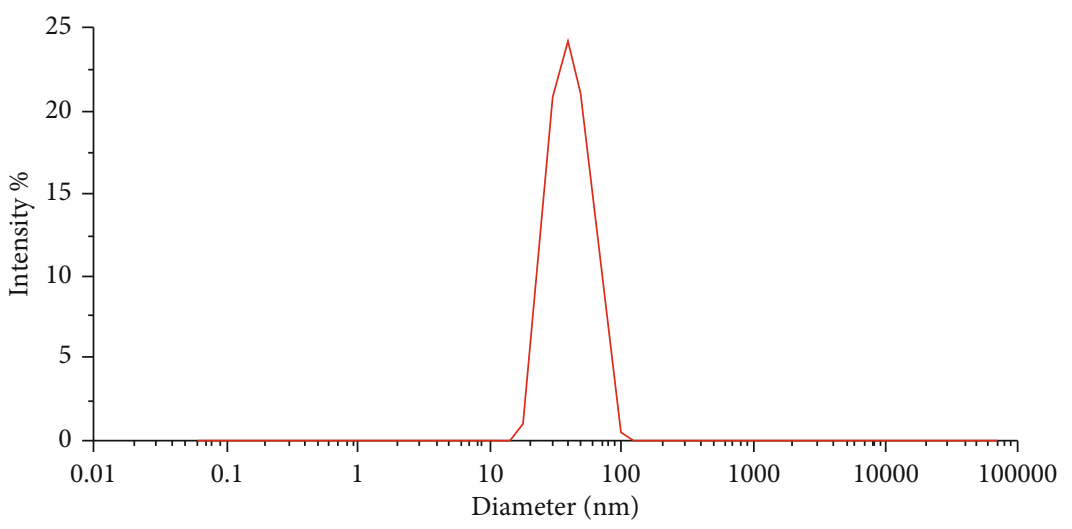

(a)

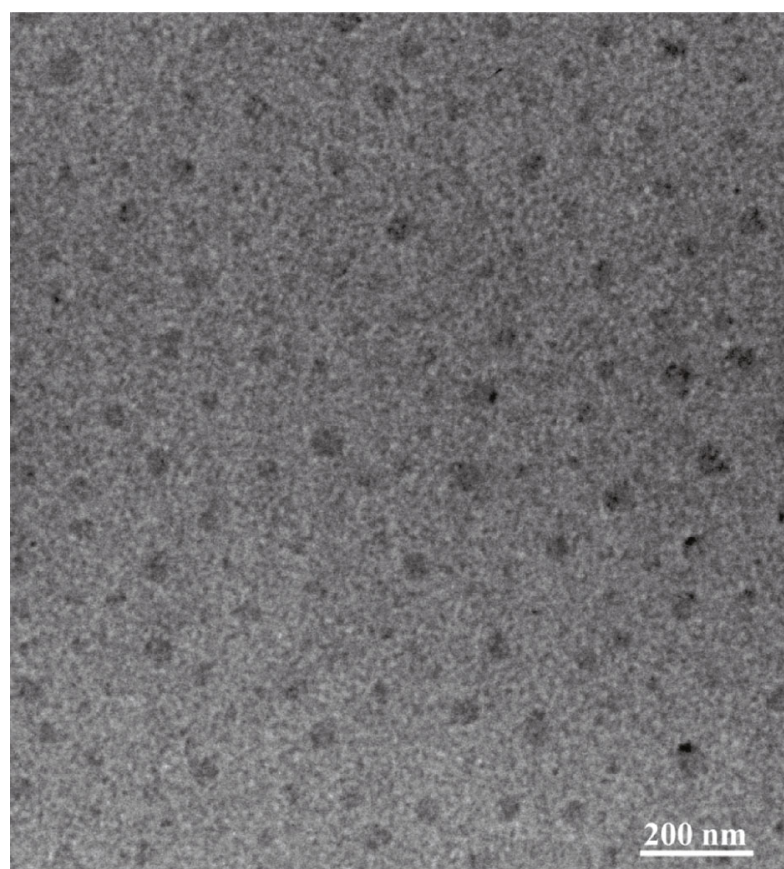

(b)

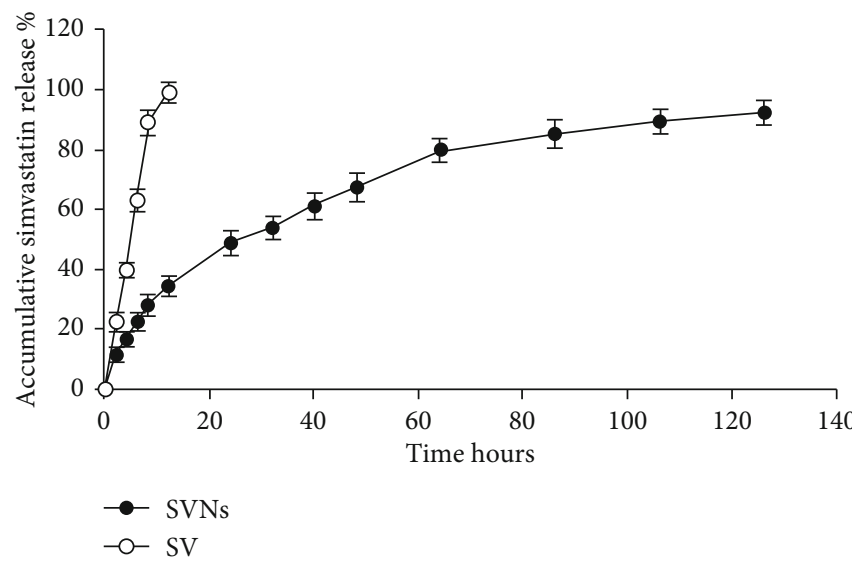

(c)

FIgURE 2: The characteristics of simvastatin-loaded nanomicelles. (a) The particle size of simvastatin-loaded nanomicelles was measured using a Zetasizer instrument. (b) Simvastatin-loaded nanomicelle morphology was observed with a transmission electron microscope (TEM). (c) The in vitro cumulative release profiles of simvastatin from simvastatin-loaded nanomicelles using human serum albumin as dissolution medium by a dynamic dialysis method. The simvastatin concentration was measured via the HPLC method at a UV absorbance of $238 \mathrm{~nm}$. The results are the mean values of three independent measurements $( \pm \mathrm{SD})$. 
ALP was normalized to the amount of total proteins synthesized.

2.2.5. ALP Staining. The slides of MG63 cells were stained for the presence of ALP using an ALP assay kit (86C-1KT; Sigma-Aldrich Corp., St. Louis, USA) according to the manufacturer's instructions after incubating in various experimental media for $7 \mathrm{~d}$. The cells were fixed in a citrateacetone-formaldehyde solution for $30 \mathrm{~s}$ and then incubated in an ALP mixture solution containing sodium nitrite solution, fast blue BB base (FBB-) alkaline solution, and naphthol AS-BI alkaline solution as a substrate for $30 \mathrm{~min}$ at room temperature away from light. Stained cells were rinsed twice with deionized water and photographed.

2.2.6. Western Blotting. MG63 cells were seeded onto 6-well plates at $5 \times 105$ cells/well and cultured with the corresponding drugs according to the experimental group. The protein expression levels of osterix (OSX) and osteocalcin (OC) were determined by western blot analysis according to the following steps: cultured cells were washed twice with ice-cold PBS, and then, the total proteins were extracted from the cells using the RIPA lysis buffer containing a protease inhibitor (Cell Signaling, Cell Signaling Technology Inc., MA, USA) and phosphatase inhibitors (Cell Signaling, Cell Signaling Technology Inc., MA, USA). The protein concentrations were determined using the bicinchoninic acid (BCA) protein assay (Pierce BCA Protein Assay Kit; Thermo Fisher Scientific). An equal amount of protein $(20 \mu \mathrm{g} / \mathrm{lane})$ was separated by $10 \%$ sodium dodecyl sulfate-polyacrylamide gel electrophoresis, then transferred to polyvinylidene difluoride (PVDF) membranes (Millipore, Bedford, MA, USA). After blocking with 5\% BSA in TBS with Tween-20 (TBST) for $60 \mathrm{~min}$, the PVDF membranes were incubated with primary antibodies at $4{ }^{\circ} \mathrm{C}$ overnight. Next, the membranes were incubated for $60 \mathrm{~min}$ at room temperature with a horseradish peroxidase-linked secondary antibody. The bands were visualized using the enhanced chemiluminescence (ECL) detection system. The quantification of protein was calculated by densitometry analysis using the ImageJ software. The primary antibodies used were specific for OSX $(1: 1500$ diluted; Abcam, Cambridge, UK), OC (1:1500 diluted; Abcam), and GAPDH (1:1500 diluted; Cell Signaling, Cell Signaling Technology Inc., MA, USA).

2.3. Animal Experiment. Six New Zealand white rabbits (2024 weeks, $2.3-0.3 \mathrm{~kg}$ ) were used as experimental animals in this experiment. The experimental protocol was approved by the Animal Ethics Committee at Shenzhen University.

2.3.1. Surgical Procedure. All surgical procedures were performed under sterile conditions. First, the animals were anesthetized with an injection of 3\% sodium pentobarbital $(30 \mathrm{mg} / \mathrm{kg})$ into the lateral ear vein. Then, the head of the rabbit was shaved and sterilized with iodine solution. Next, $2 \mathrm{ml}$ of $2 \%$ lidocaine was administered to the operating site as local anesthesia. A perpendicular incision was made, and a mucoperiosteal flap was raised. Four critical bone defects $(6.5 \mathrm{~mm}$ in diameter) were created at the top of the skull and randomly divided into the following four groups: no augmenta-

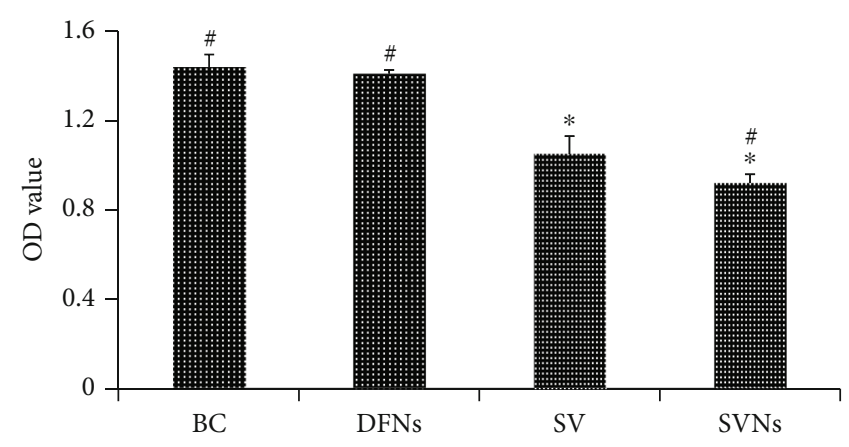

FIgURE 3: The effect of SVNs and SV on the proliferative activity of MG63 cells. The results are the mean values of three independent measurements $( \pm \mathrm{SD}) .{ }^{*} P<0.05$ vs. BC group; ${ }^{\#} P<0.05$ vs. SV group.

tion (blank control, BC) group, gelatin sponge (GS) group, gelatin sponge with $0.5 \mathrm{mg} \mathrm{SV}$ (SV-GS) group, and gelatin sponge with $5 \mathrm{mg}$ SVNs ( $0.5 \mathrm{mg}$ SV) (SVNs-GS) group. The surgical procedure is depicted in Figure 1. The animals were sacrificed four weeks after surgery.

2.3.2. Microcomputed Tomography (Micro-CT) Analysis. The effects of SVNs and SV on osteogenesis were evaluated radiographically. The specimens were removed en bloc, fixed in $10 \%$ neutral formalin, and scanned with a micro-CT scanner (Skyscan-1176; Bruker, Belgium) (pixel size: $18.05 \mu \mathrm{m}$; source voltage: $62 \mathrm{kV}$; source current: $380 \mu \mathrm{A}$ ). Micro-CT images were reconstructed using Skyscan NRecon software (Bruker, Belgium). The bone defect area $(6.5 \mathrm{~mm}$ in diameter) of each image was defined as the region of interest (ROI), and the volume of interest (VOI) was made up of the ROIs of 150 images. The newly formed bone volume $(\mathrm{BV})$, bone mineral density (BMD), percent bone volume (bone volume/tissue volume, BV/TV), and trabecular number (Tb.N) of the newly formed bone were measured using Skyscan CTAn software (Bruker, Belgium).

2.3.3. Histomorphometric Analysis. After the specimens were completely fixed, they were demineralized with $10 \%$ EDTA for four weeks and paraffin-embedded after dehydration. All the specimens were sectioned into $4 \mu \mathrm{m}$-thick serial sections for hematoxylin and eosin (HE) and Masson's trichrome staining for observation of the newly formed bone under a microscope.

2.4. Statistical Analysis. Statistical analysis was performed using SPSS version 19.0 (IBM Corp., Armonk, NY, USA). All data are shown as the mean \pm standard deviation (SD) from at least three independent experiments and were analyzed by repeated-measures ANOVA and Student's $t$-test.

\section{Results}

3.1. Characterization of Simvastatin-Loaded Nanomicelles. The mean diameter of the SVNs was determined with dynamic light scattering (DLS) and found to be approximately $38.97 \pm 1.96 \mathrm{~nm}$ (Figure 2(a)). The SVNs were nearly spherical in shape based on the TEM results (Figure 2(b)). 


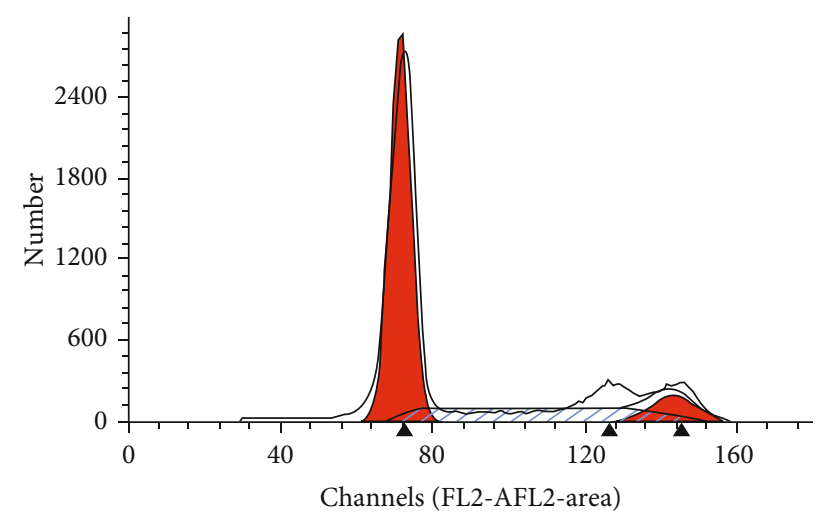

BC

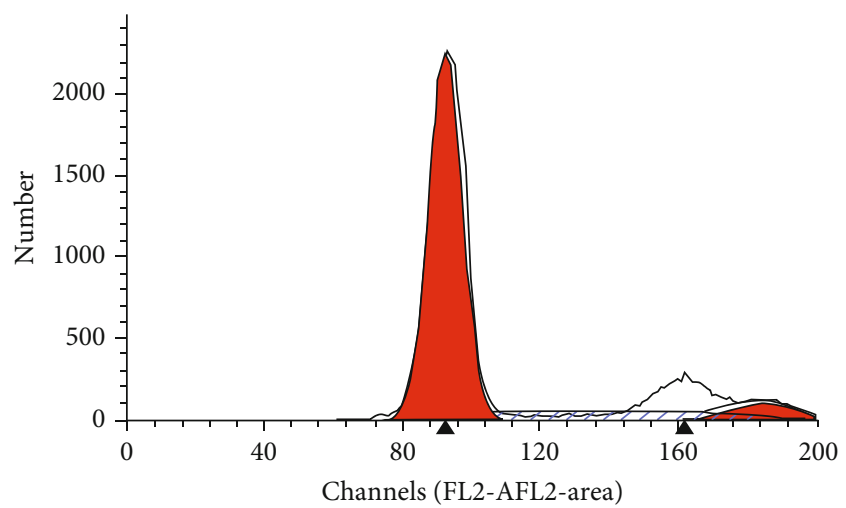

SV
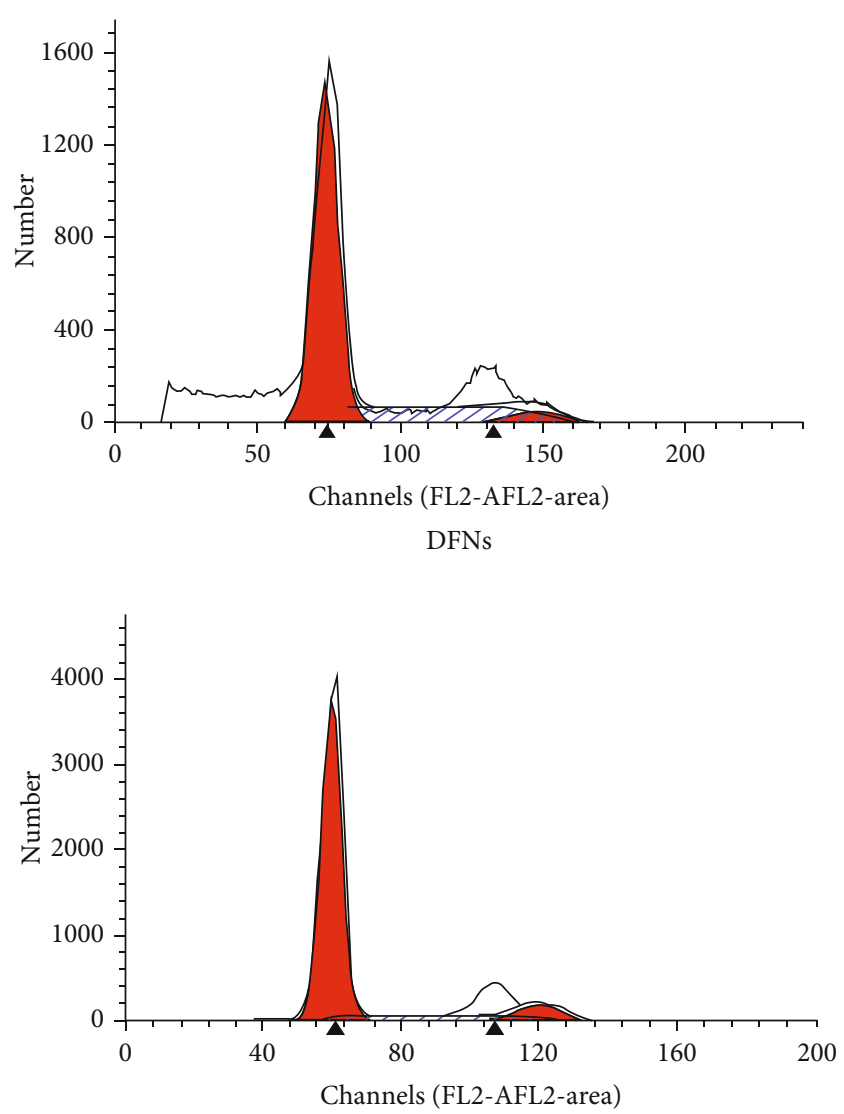

SVNs

Dip G0-G1

Dip G2-M

', Dip S

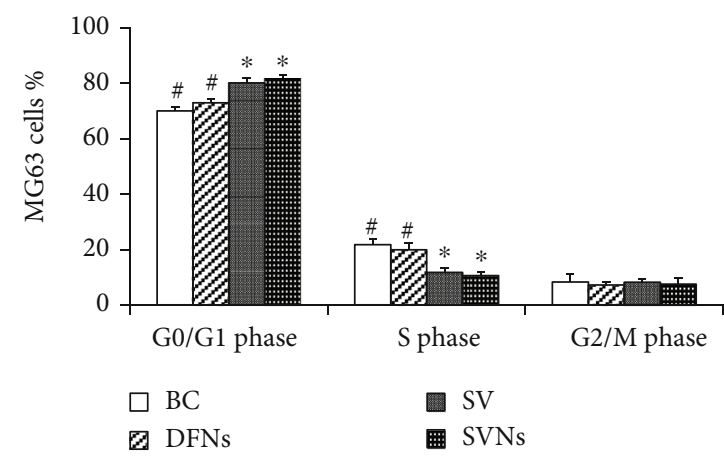

FIGURE 4: The effect of SVNs and SV on the cell cycle of MG63 cells. The results are the mean values of three independent measurements $( \pm \mathrm{SD}) .{ }^{*} P<0.05$ vs. BC group; ${ }^{\#} P<0.05$ vs. $\mathrm{SV}$ group.

Under 20\% theoretical drug-loading capacity (DLC) conditions, the SVNs presented an encapsulation ratio and drugloading efficiency of $54.57 \pm 3.15 \%$ and $10.91 \pm 0.63 \%$, respectively. These nanomicelles were selected for subsequent experiments to evaluate their osteogenic effects.

3.1.1. In Vitro Simvastatin Release. As shown in Figure 2(c), the free SV was nearly completely released at $12 \mathrm{~h}$, whereas only approximately $35 \%$ of the total SV had been released from the nanomicelles at the same time. SVNs showed good sustained-release properties in vitro.

\subsection{Cell Experiment Results}

3.2.1. Cell Proliferation. Figure 3(a) shows that the number of cells significantly decreased in the SVNs group and the SV group at $3 \mathrm{~d}$ after administration of the drugs in comparison with the BC group. The SVNs group had fewest cells, 

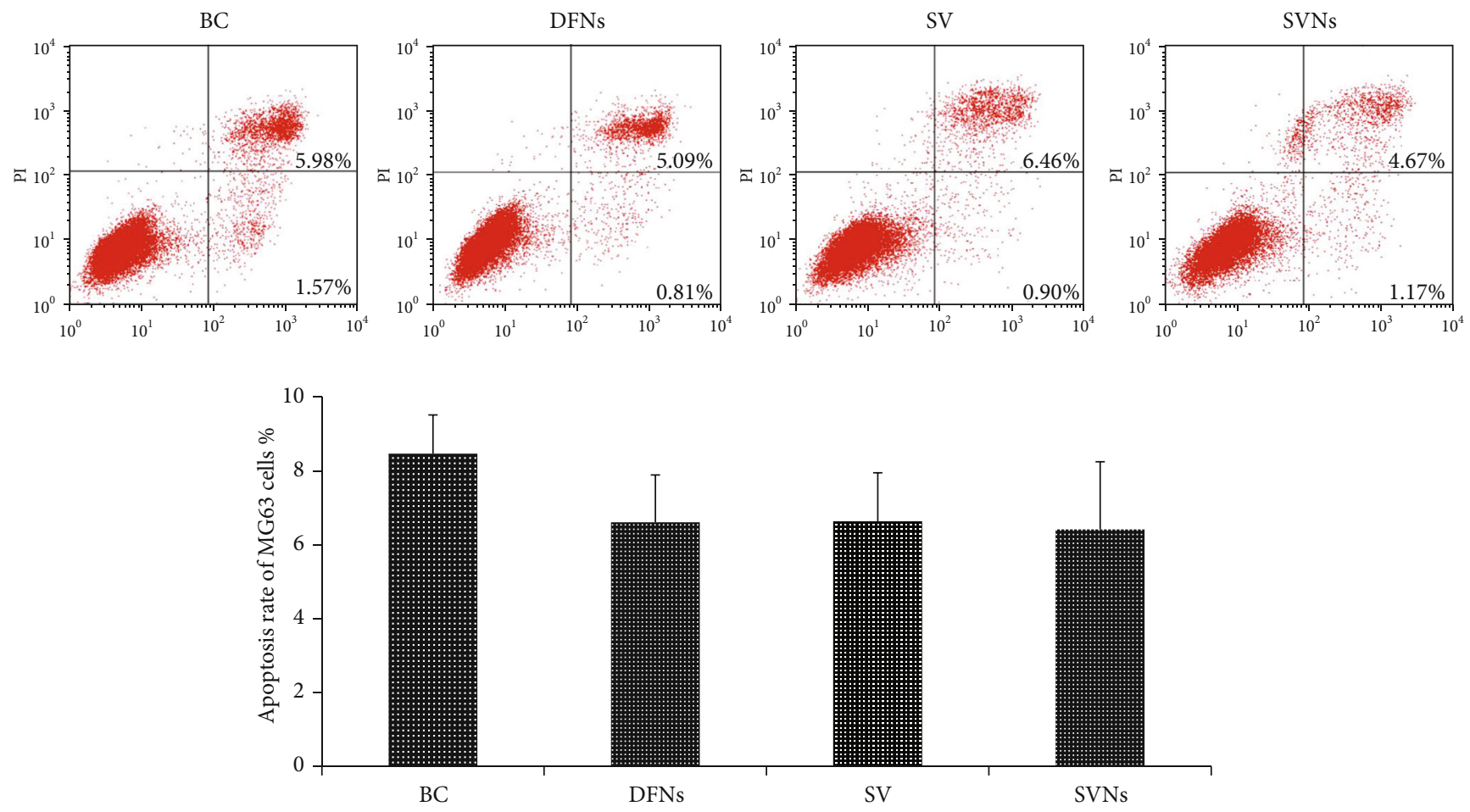

FIGURE 5: The effect of SVNs and SV on the apoptotic rate of MG63 cells. The results are the mean values of three independent measurements $( \pm \mathrm{SD}) .{ }^{*} P<0.05$ vs. BC group; ${ }^{\#} P<0.05$ vs. SV group.

indicating that SVNs exerted a significantly more potent inhibitory effect on cell proliferation than SV.

3.2.2. Cell Cycle and Cell Apoptosis. To clarify whether the inhibitory effect of SVNs and SV on the proliferative activity of MG63 cells is related to a drug-induced alteration of cell cycle and apoptotic rate, flow cytometry was used to analyze the cell cycle and apoptotic rate of the MG63 cells in all experimental groups.

Figure 4 shows that the percentages of the MG63 cells in the G0/G1 phases of the cell cycle were significantly increased, while the percentage of cells in the $S$ phase of the cell cycle was markedly reduced, in the SVNs group and the SV group $3 \mathrm{~d}$ after administration of the drugs in comparison with the $\mathrm{BC}$ group. No significant changes were observed in the percentages of $\mathrm{G} 2 / \mathrm{M}$ phase cells.

Moreover, Figure 5 shows that the apoptotic rate of MG63 cells did not increase significantly after treatment with SVNs and SV. The above cell proliferation experiment results show that SVNs and SV significantly reduced the number of MG63 cells. The decrease in the number of cells in the SVNs group and the SV group did not appear to be related to apoptosis.

3.2.3. ALP Activity Assay. The osteoblastic differentiation of MG63 cells was evaluated by measuring the ALP activity after culturing with different drug formulations for $7 \mathrm{~d}$. As shown in Figure 6(a), the ALP activities of cells in the SVNs group and SV group were significantly higher than those of cells in the BC group, and the SVNs group exhibited the greatest cellular ALP activity. ALP staining results (Figure 6(b)) showed obvious blue areas in the SVNs group and SV group, especially in the SVNs group, and almost no blue areas were observed in the DFN group and BC group. These results are consistent with those of the quantitative analysis of ALP activity described above.

3.2.4. Protein Levels of OSX and OC. The protein expression levels of OSX and OC in MG63 cells were determined by the western blot analysis. At $7 \mathrm{~d}$ or $14 \mathrm{~d}$ after drug application, the protein expression levels of OSX (Figure 6(c)) and OC (Figure 6(d)) of cells in the SVNs group and SV group were significantly higher than those of the cells in the BC group. SVNs could further significantly enhance the promoting effect of SV on the expression of OSX or OC in MG63 cells.

The above results showed that SVNs and SV significantly promoted the osteogenic differentiation ability of the MG63 cells in vitro. Their osteogenesis-promoting effect in vivo requires further verification in animal experiments.

3.3. Animal Experiment Results. The animal experiment was executed smoothly, the experimental animals healed well after surgery, and no complications such as inflammation, infection, splitting, or exposure were noted in the surgical area. During specimen preparation, one specimen was damaged and was not included in the subsequent analysis. The number of samples in each group was $n=5$.

3.3.1. Microcomputed Tomography (CT) Analysis Results. Animals were sacrificed by air injection into an ear vein 4 weeks after surgery. Under sterile conditions, the surgical 


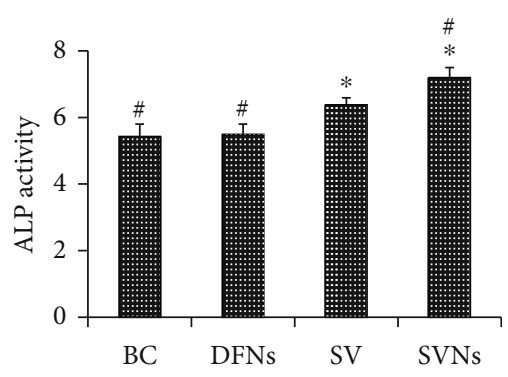

(a)
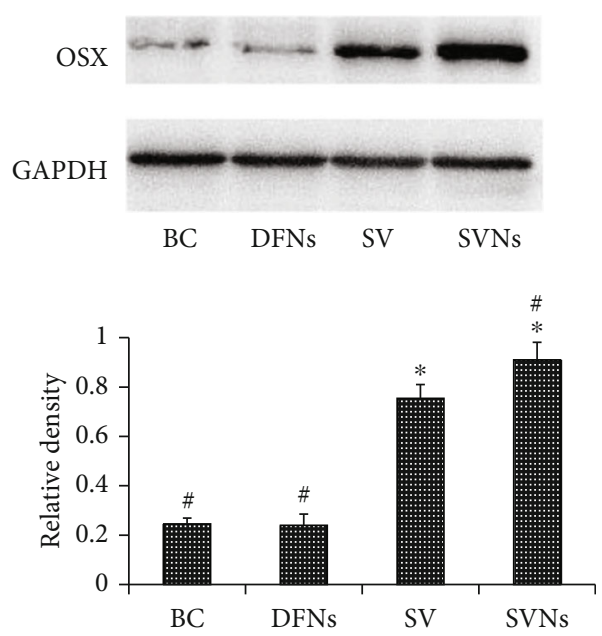

(c)

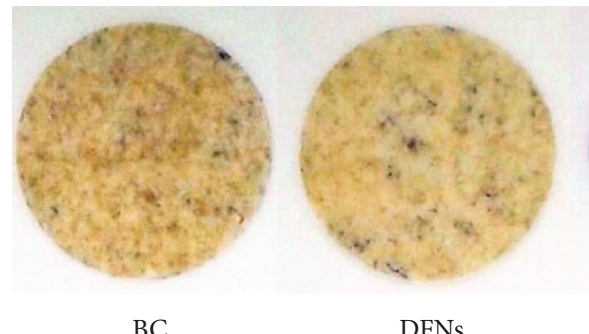

$\mathrm{BC}$

DFNs

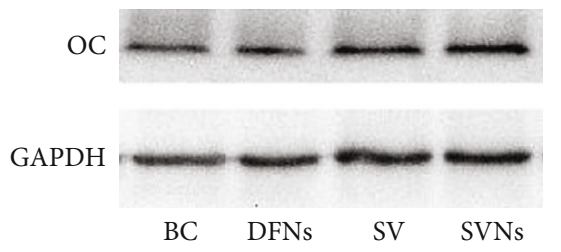

(b)
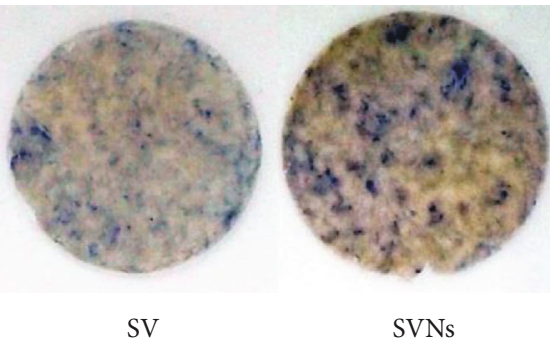

SVNs

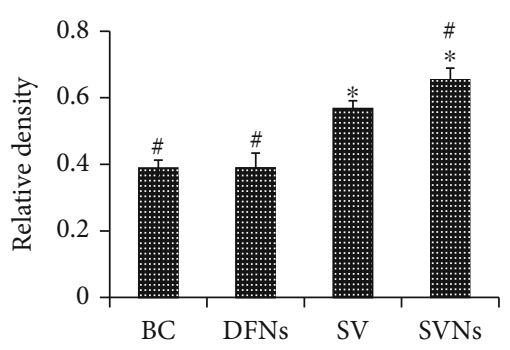

(d)

FIGURE 6: The effect of SVNs and SV on the osteogenic differentiation of MG63 cells. (a) ALP activity was measured in whole-cell extracts. (b) ALP staining. The ALP activity was proportional to the intensity of the blue-violet color. (c) The protein expression of OSX after treatment with different drug formulations for $7 \mathrm{~d}$. (d) The protein expression of OC after treatment with different drug formulations for $14 \mathrm{~d}$. The results are the mean values of three independent measurements $( \pm \mathrm{SD}) .{ }^{*} P<0.05$ vs. BC group; ${ }^{\#} P<0.05$ vs. SV group.

area was completely delineated at the site larger than $5 \mathrm{~mm}$ along the edge of the defect area. After scanning with micro-CT, the Skyscan CTAn software of the scanner was used to perform three-dimensional reconstruction of the defect area (Figure 7(a)). At 4 weeks after surgery, each experimental group had different degrees of new bone formation, and the direction of the new bone formation mainly extended from the edge of the bone defect toward the center of the defect area. New bone formation was most extensive in the SVNs-GS group, followed by the SV-GS group, while new bone formation was the least obvious in the $\mathrm{BC}$ group, and large defects were still present in the bone defect area. The bone defect area was further divided into VOI, and threedimensional reconstructed images more clearly showed that the new bone formation in the SVNs-GS group was significantly more obvious than that in the other groups (Figure 7(b)). Quantitative analysis of BMD (Figure 7(c)), BV/TV ratio (Figure $7(\mathrm{~d})$ ), and Tb.N (Figure $7(\mathrm{e})$ ) in the new bone by Skyscan CTAn software showed that the BMDs in the SVNs-GS, SV-GS, and GS groups were $362.1 \%$, $292.0 \%$, and $183.5 \%$ of the BMD in the BC group; the BV/TV ratios were $181.3 \%, 158.0 \%$, and $133.8 \%$ of the $\mathrm{BV} / \mathrm{TV}$ ratio in the $\mathrm{BC}$ group; and the Tb.N values were $215.2 \%, 181.8 \%$, and $145.4 \%$ of the Tb.N in the BC group, respectively.
3.3.2. Histological Evaluation Results. At 4 weeks after surgery, sagittal primary bone slices obtained from the centers of the bone defect areas were subjected to HE staining (Figure 8). The results showed that the bone defect areas of each group were covered by new fibrous tissue and immature bone tissue, with a small amount of irregular cancellous bone at the edge of the primary bone defect. The SVNs-GS group had the most newly formed irregular cancellous bone and the smallest remaining bone defect areas. The new bone formation in the SVNs-GS group and SV-GS group was significantly greater than that in the BC group and GS group. Under high magnification, the new bone tissue in each group was identified as irregular fibrous bone, and the arrangement of trabecular bone was disordered; a large number of spindleshaped osteoblasts were attached to the surface of the primary trabecular bone, and some osteoblasts had transformed into immature bone cells and were buried in the bone lacunae. The extensive new capillary formation was evident around the primary trabecular bone, especially in the SVNs-GS group.

Adjacent blank sections were subjected to Masson trichrome staining (Figure 9). The results showed that the new fibrous tissues in the bone defect areas of all groups were stained blue, the new bone tissue at the edge of the bone 


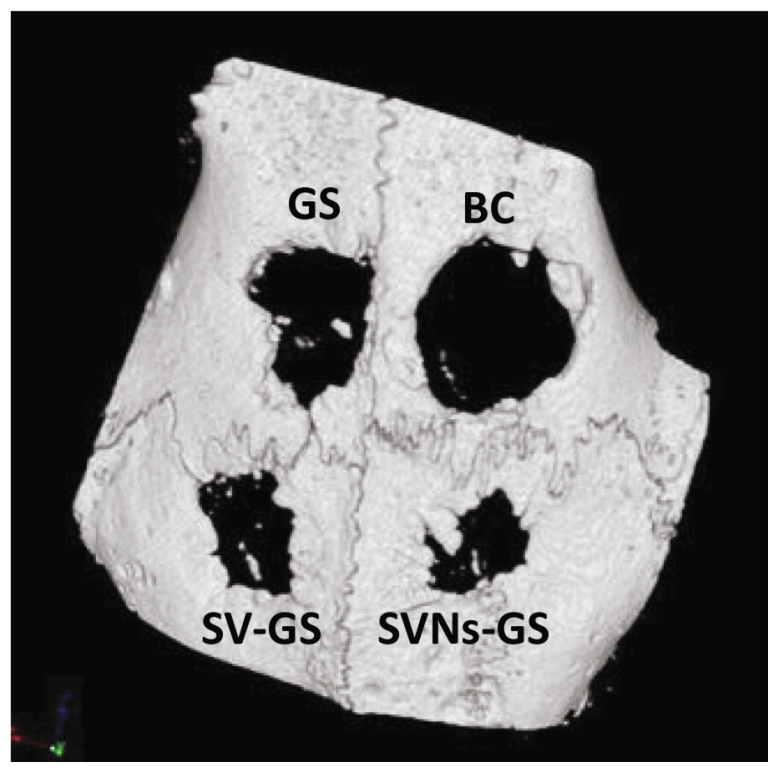

(a)
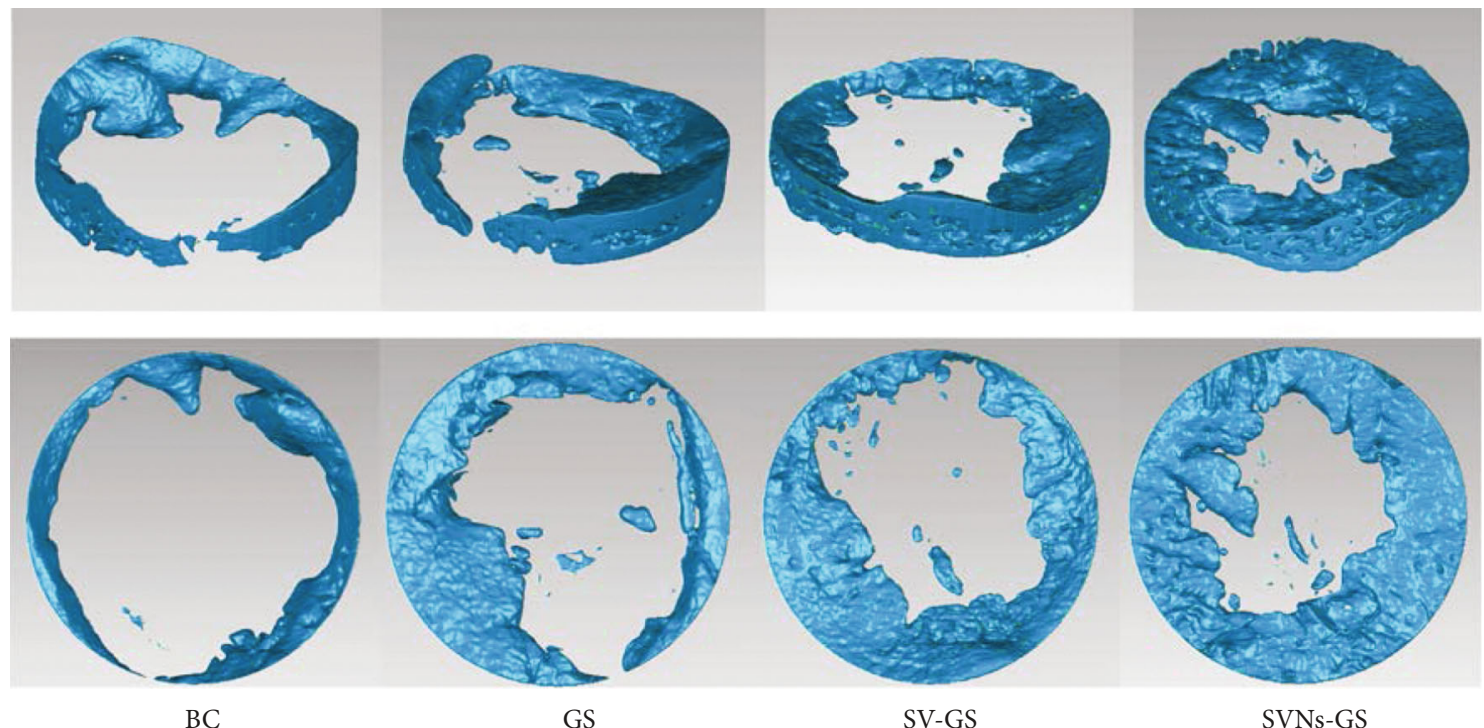

(b)

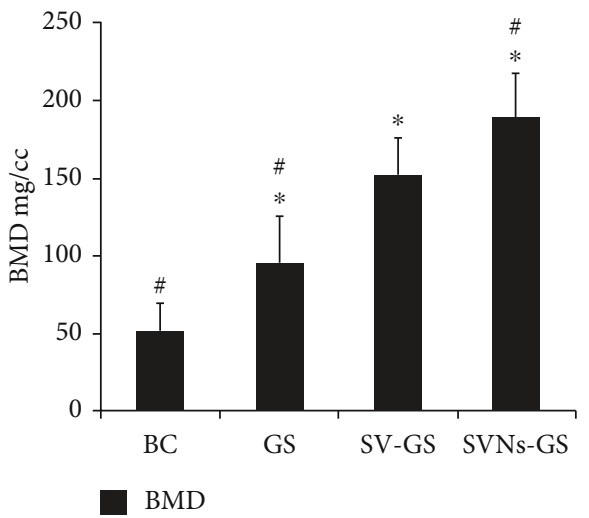

(c)

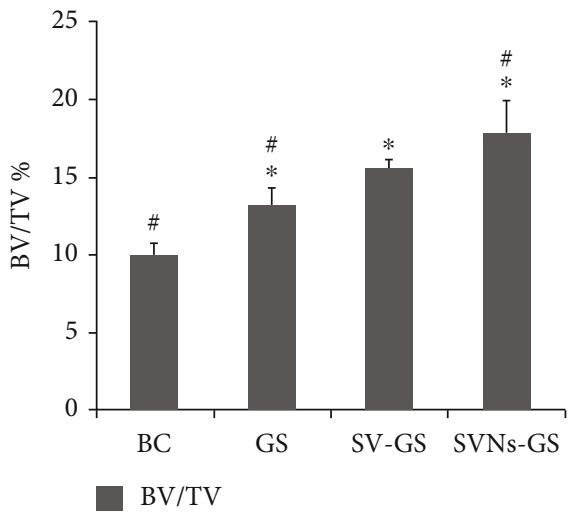

(d)

Figure 7: Continued. 


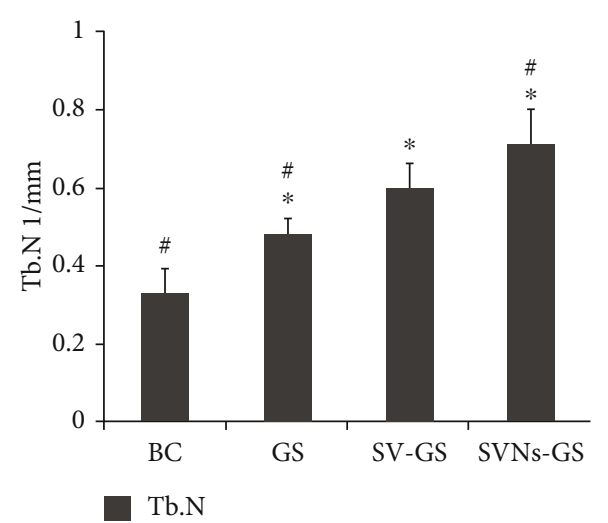

(e)

FIGURE 7: Evaluation of new bone formation in the surgical defect area with micro-CT scanning at 4 weeks after surgery. (a) Threedimensional reconstruction of the defect area. (b) Three-dimensional reconstruction of VOI. Quantitative analysis of BMD (c), BV/TV ratio (d), and Tb.N (e) in the new bone. The results are the mean values of five independent measurements $( \pm \mathrm{SD}) .{ }^{*} P<0.05$ vs. $\mathrm{BC}$ group; ${ }^{\#} P<0.05$ vs. SV group.

defect area was stained blue or red and blue, and the surrounding old bone was stained red. In the BC and GS groups, the arrangement of collagen fibers in the new fibrosis tissues, which showed lighter staining, was sparse and slightly disordered; only a small amount of new bone formation was noted on the edge of the defect, the bone defect area was large, trabecular bone was scarce, and no obvious mature red lamellar bone tissue was observed. In the SVNs-GS and SV-GS groups, the arrangement of collagen fibers in the new fibrosis tissues, which showed darker staining, was regular and dense; a large number of blue or red-blue primary trabecular bones had formed at the edge of the defect area and extended to the center of the defect area, the bone defect area was significantly reduced, and the trabecular bones at the proximal end of the bone defect appeared to be almost mature and exhibited red-stained lamellar bone. These observations were especially obvious in the SVNs-GS group. Under high magnification, the SVNs-GS group and SV-GS group had a large amount of osteoblasts with blue-violet nuclei attached to new trabecular bones that were gradually surrounded by newly formed osteoid, which were buried inside a new trabecular bone to form lacunae, and osteoblasts had gradually transformed into bone cells. The BC and GS groups had formed few new trabecular bones, osteoblasts were mainly located in the newly formed collagen fibers, and no apparent mature bone tissue structure was noted.

\section{Discussion}

Autogenic bone, allogeneic bone, and artificial bone replacement materials are often used in the repair of large bone defects [3-7]. Adding exogenous osteogenic growth factors such as BMP-2 to these materials can increase the osteoinductivity of the materials, thereby enhancing the osteogenesispromoting effect [11-14]. However, the high cost and short half-life of exogenous osteogenic growth factors largely limit their clinical application [15]. SV, a commonly used lipidlowering agent, has been found to increase the expression of BMP-2 in osteoblasts in vivo to promote the formation of new bone in bone defect areas $[28,36]$. Therefore, $\mathrm{SV}$ is expected to replace exogenous BMP-2 for bone formation promotion since the cost of SV is 16,000-times lower than that of BMP-2 [17].

The local concentration of SV in the treatment area is closely related to whether SV can exert its osteogenesispromoting effect. In animal experiments, SV is mainly administered systemically, and oral administration is the most common route of administration [28]. SV is a lipidsoluble drug with a low affinity for bone. After oral administration, most drugs will be metabolized by the liver, and eventually, only very small amounts of drugs can reach the treatment area [27]. The low concentration of SV in the treatment area results in a negligible promoting effect on new bone formation. Increasing the oral dose and repeated drug administration can enhance the osteogenic effect of SV. However, an excessive dose also substantially increases the likelihood of systemic side effects [28, 29]. Local administration of SV is an effective approach to avoid the above problems $[28,37]$.

Local administration of SV has the advantages of avoiding liver metabolism and improving the bioavailability of SV. Many methods can be used for local SV administration [38-40]. The simplest method is to combine SV directly with bone scaffold materials. The disadvantage of this method is that due to the burst release effect, a large amount of SV can be released from the stent into the blood and be quickly cleared from the body [28]. As a result, SV can act only for a short time, resulting in a limited osteogenic effect. Therefore, the goal of local SV administration should be slow and sustained drug release to the bone defect area to promote bone formation and to effectively improve the bioavailability of SV [40].

In previous studies, some scholars synthesized SV-loaded microspheres or nanospheres and confirmed that SV could be slowly released from the microspheres or nanospheres [41-44]. The present study used mPEG-PLA polymeric nanomicelles to encapsulate SV using the preferred dialysis method developed in a previous study. The prepared SVNs 


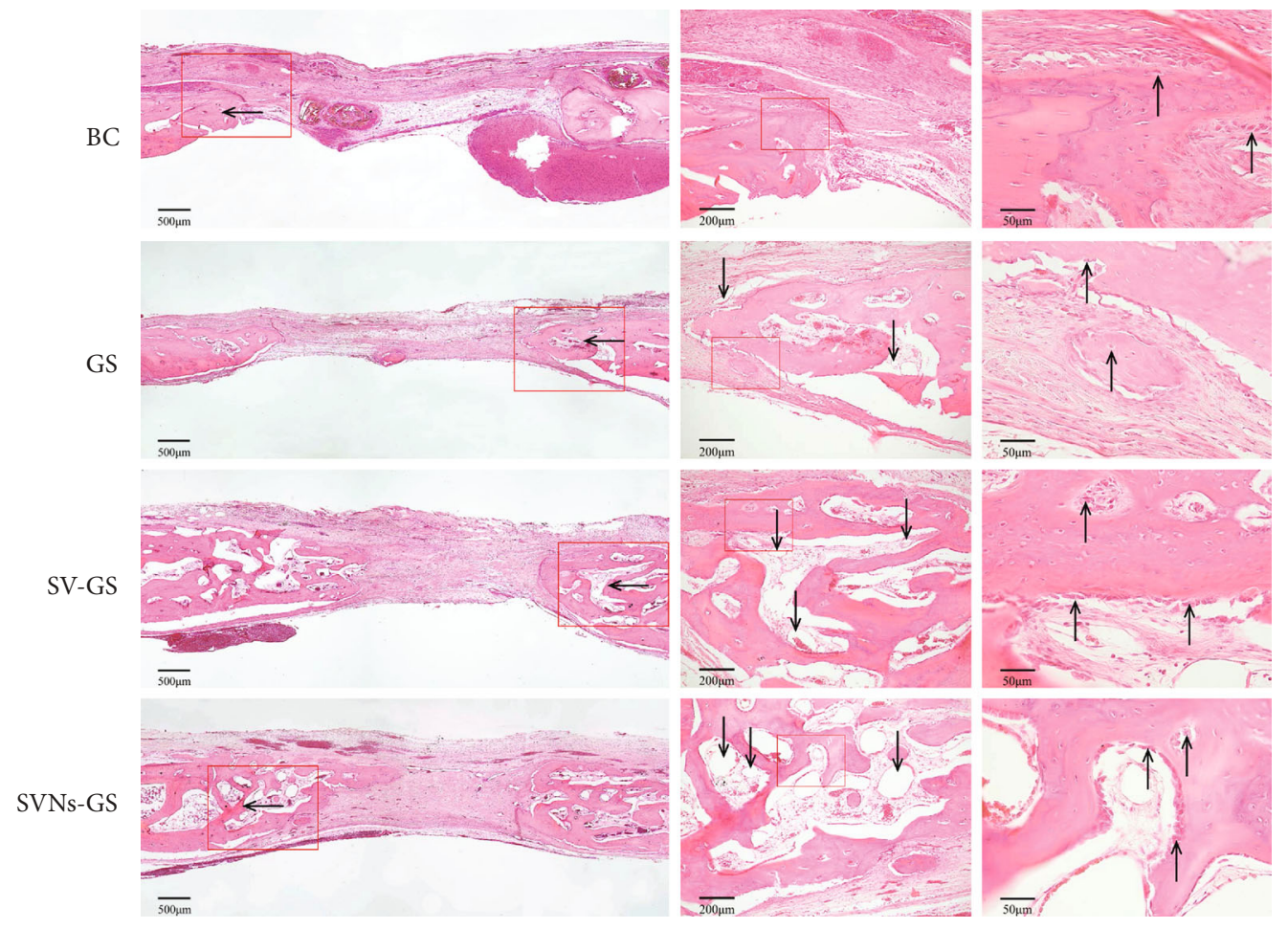

FIGURE 8: Histologic images of the surgical defects at four weeks (HE stain, $\times 40, \times 100, \times 400$ ). The “ $\leftarrow$ ” arrows point to the original defect margins, the " $\downarrow$ " arrows indicate newly formed capillary, and the " $\uparrow$ " arrows indicate osteoblasts.

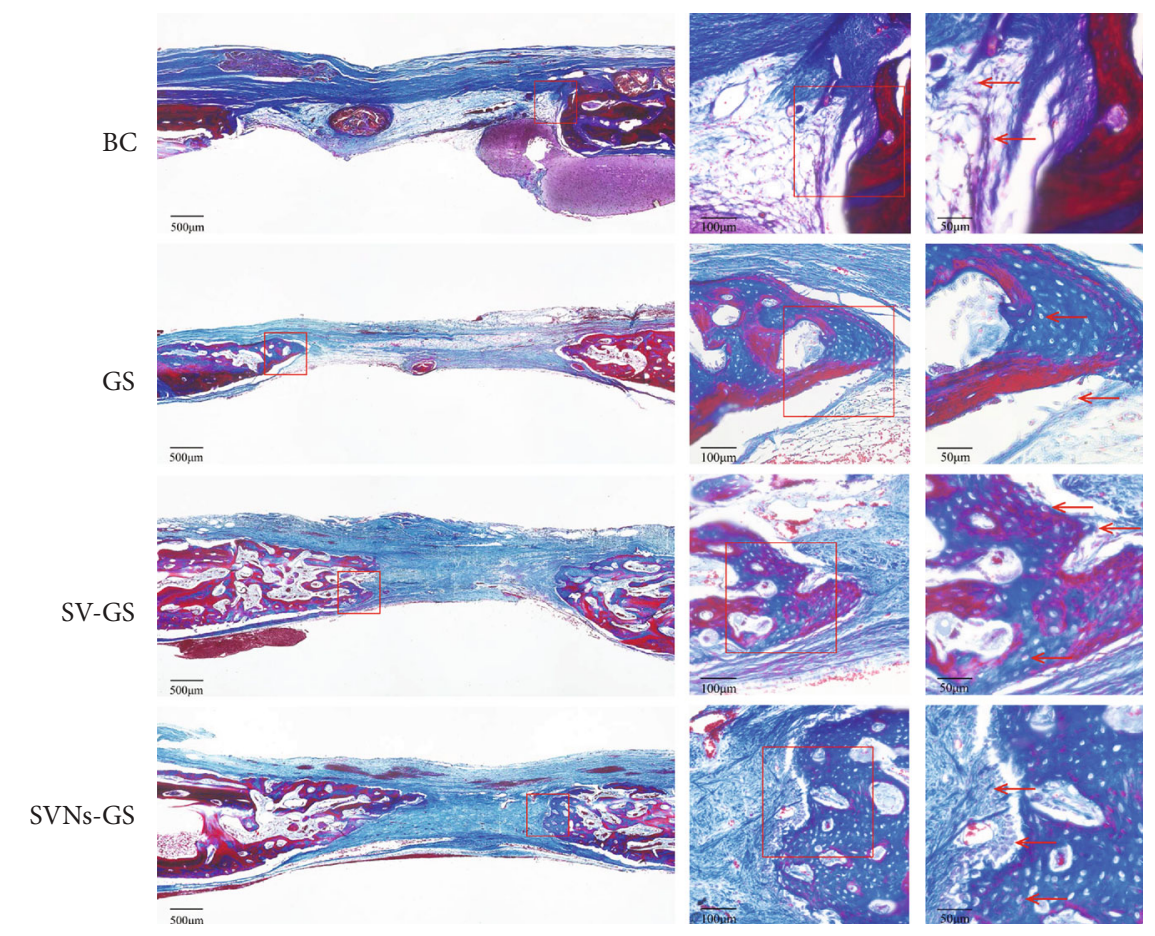

Figure 9: Histologic images of the surgical defects at four weeks (Masson stain, $\times 40, \times 100, \times 400$ ). The “ $\leftarrow$ ” arrows indicate osteoblasts.

had a small size and a spherical shape, which extended the release time of the same amount of SV from $12 \mathrm{~h}$ to over $100 \mathrm{~h}$, reflecting excellent sustained-release properties.

Few studies related to the osteogenesis-promoting effect of SV are available. Existing studies have shown that nano-based drug carriers can significantly improve the osteogenesispromoting effect of SV in vivo and in vitro [41-44]. Our previous in vitro study preliminarily confirmed these findings [24]. In the present study, we detected the ALP activity and protein expression levels of OSX and OC in MG63 cells and 
again confirmed that SVNs can significantly improve the osteoblast differentiation-promoting effect of SV in vitro. In previous studies, we found that after adding SVNs and SV, the number of MG63 cells was significantly decreased compared with that in the control group, and cell proliferation activity was inhibited. The premise of cell differentiation is currently believed to be that cells stop proliferation. Whether the reduced number of cells after drug application is the result of cell differentiation or drug-induced apoptosis requires further investigation. The results of the present study showed that SVNs and SV can block MG63 cells in the G0/G1 phase and thus inhibit cell proliferation; however, SVNs and SV did not increase the cell apoptosis rate. These results suggest that the osteoblast differentiation-promoting effects of SVNs and SV are closely related to changes in the osteoblast cell cycle.

We also evaluated the in vivo osteogenic effects of SVNs and SV through a rabbit skull defect model. To avoid the drawbacks of systemic administration, local SV administration was used in the study. Because micro-CT scans of specimens were needed in the later stage to observe and analyze the new bone formation, we loaded SVNs and SV on gelatin sponges, which do not interfere with X-rays. The amount of SV used in the animal experiments had a significant impact on the experimental results. SV at a low concentration cannot promote the formation of new bone, while excessively high doses of SV can trigger inflammation in the bone defect area and affect bone healing [45]. In animal experiments, doses of $0.1 \mathrm{mg}$ to $0.5 \mathrm{mg}$ of SV have been suggested to be suitable [46]. Within this range, the bone healing-promoting effect of SV was directly proportional to the amount of SV used $[47,48]$. Therefore, we selected $0.5 \mathrm{mg}$ as the dose of SV in the present study. Micro-CT quantitative analysis results showed that SVNs and SV both significantly promoted the formation of new bone and significantly increased the BMD and the amount of new trabecular bone in the bone defect area. It is well known that the formation of new bone mainly depends on the number of osteoblasts. Histomorphology qualitative results showed that both SVNs and SV significantly increased the number of osteoblasts in the bone defect area. In addition, both SVNs and SV increased the number of new blood vessels in the bone defect area, which may be related to the promotion of the expression of vascular endothelial growth factor by SVNs and SV, thus stimulating the differentiation of endothelial progenitor cells and ultimately stimulating angiogenesis. The increases in the number of osteoblasts and new blood vessels both promoted the formation and maturation of new bones in the bone defect area. The above quantitative and qualitative results showed that compared with the same amount of SV, SVNs had a greater effect on the promotion of new bone formation in the bone defect area, which may be due to the slow and sustained release of SV by SVNs. SVNs continuously and slowly release SV into the bone defect area to maintain a high local concentration while substantially prolonging the duration of SV action, resulting in a stronger osteogenic effect. In addition, due to their small size, nano-based drug carriers have physical and chemical properties (such as surface effects and small-size effects) that traditional materials do not have. Therefore, SVNs and SV may have significantly different effects on cellular uptake, intracellular distribution, and regulation of osteogenesis-related signaling pathways. Compared with SV, SVNs have a stronger osteogenic effect.

This study has certain limitations. This study did not elucidate the possible mechanism through which SVNs significantly improve the in vivo and in vitro osteogenesispromoting effect of SV. In addition, this study mainly focused on the effects of SVNs and SV on the early stage of osteogenesis, and further studies are needed to investigate their roles in the late stage of osteogenesis. Finally, porous scaffolds play an important role in bone tissue engineering [49]. In the future, we will try to prepare porous scaffolds combined with SVNs in order to achieve better bone defect repair effects.

\section{Conclusion}

This study showed that polymeric nanomicelles can be used to encapsulate SV to prepare SVNs. In vitro, SVNs can promote the differentiation of osteoblasts by arresting osteoblasts at the G0/G1 phase and significantly increase the ALP activity and protein expression levels of markers associated with osteogenesis. In vivo, SVNs can promote the formation and mineralization of new bones in bone defect areas by increasing the number of osteoblasts and new blood vessels and accelerating the repair process of bone defects. Therefore, polymeric nanomicelles can be used as SV carriers to enhance the osteogenic effect of SV.

\section{Data Availability}

The data used to support the findings of this study are available from the corresponding author upon request.

\section{Conflicts of Interest}

The authors report no conflicts of interest in this work.

\section{Authors' Contributions}

Xianling Feng and Xinxin Yue contributed equally to this article.

\section{Acknowledgments}

This work was supported by grants from the Characteristic Innovation Project of Guangdong Province (No. 2018GKTSCX028), the Medical Science and Technology Research Foundation of Guangdong Province (No. A2018170), and the Innovative Engineering Project of Shenzhen Polytechnic (cxgc2019c0004).

\section{References}

[1] A. A. El-Rashidy, J. A. Roether, L. Harhaus, U. Kneser, and A. R. Boccaccini, "Regenerating bone with bioactive glass scaffolds: a review of in vivo studies in bone defect models," Acta Biomaterialia, vol. 62, pp. 1-28, 2017.

[2] A. Noori, S. J. Ashrafi, R. Vaez-Ghaemi, A. Hatamian-Zaremi, and T. J. Webster, "A review of fibrin and fibrin composites for 
bone tissue engineering," International Journal of Nanomedicine, vol. Volume 12, pp. 4937-4961, 2017.

[3] A. R. Amini, C. T. Laurencin, and S. P. Nukavarapu, "Bone tissue engineering: recent advances and challenges," Critical Reviews in Biomedical Engineering, vol. 40, no. 5, pp. 363408, 2012.

[4] Z. P. Du, X. X. Feng, G. X. Cao et al., "The effect of carbon nanotubes on osteogenic functions of adipose-derived mesenchymal stem cells in vitro and bone formation in vivo compared with that of nano-hydroxyapatite and the possible mechanism," Bioactive Materials, vol. 6, no. 2, pp. 333-345, 2021.

[5] Y. Li, S. K. Chen, L. Li, L. Qin, X. L. Wang, and Y. X. Lai, "Bone defect animal models for testing efficacy of bone substitute biomaterials," Journal of Orthopaedic Translation, vol. 3, no. 3, pp. 95-104, 2015.

[6] A. Bow, D. E. Anderson, and M. Dhar, "Commercially available bone graft substitutes: the impact of origin and processing on graft functionality," Drug Metabolism Reviews, vol. 51, no. 4, pp. 533-544, 2019.

[7] D. J. Hall, T. M. Turner, and R. M. Urban, "Healing bone lesion defects using injectable CaSO4/CaPO4 -TCP bone graft substitute compared to cancellous allograft bone chips in a canine model," Journal of Biomedical Materials Research. Part B, Applied Biomaterials, vol. 107, no. 2, pp. 408-414, 2019.

[8] A. Kadam, P. W. Millhouse, C. K. Kepler et al., "Bone substitutes and expanders in spine surgery: a review of their fusion efficacies," International Journal of Spine Surgery, vol. 10, p. 33, 2016.

[9] T. Rolvien, M. Barbeck, S. Wenisch, M. Amling, and M. Krause, "Cellular mechanisms responsible for success and failure of bone substitute materials," International Journal of Molecular Sciences, vol. 19, no. 10, p. 2893, 2018.

[10] M. Yamada and H. Egusa, "Current bone substitutes for implant dentistry," Journal of Prosthodontic Research, vol. 62, no. 2, pp. 152-161, 2018.

[11] Z. P. Du, G. X. Cao, K. Li, R. H. Zhang, and X. M. Li, "Nanocomposites for the delivery of bioactive molecules in tissue repair: vital structural features, application mechanisms, updated progress and future perspectives," Journal of Materials Chemistry B, 2020.

[12] A. Decambron, A. Fournet, M. Bensidhoum et al., "Low-dose BMP-2 and MSC dual delivery onto coral scaffold for critical-size bone defect regeneration in sheep," Journal of Orthopaedic Research, vol. 35, no. 12, pp. 2637-2645, 2017.

[13] M. Koolen, A. Longoni, J. van der Stok, O. Van der Jagt, D. Gawlitta, and H. Weinans, "Complete regeneration of large bone defects in rats with commercially available fibrin loaded with BMP-2," European Cells \& Materials, vol. 38, pp. 94105, 2019.

[14] L. Wei, F. Teng, L. Deng et al., "Periodontal regeneration using bone morphogenetic protein 2 incorporated biomimetic calcium phosphate in conjunction with barrier membrane: a pre-clinical study in dogs," Journal of Clinical Periodontology, vol. 46, no. 12, pp. 1254-1263, 2019.

[15] P. J. Boyne, S. Salina, A. Nakamura, F. Audia, and S. Shabahang, "Bone regeneration using rhBMP-2 induction in hemimandibulectomy type defects of elderly sub-human primates," Cell and Tissue Banking, vol. 7, no. 1, pp. 1-10, 2006.

[16] J. Lewandowski, M. Sinski, L. Puchalska, B. Symonides, and Z. Gaciong, "Simvastatin but not ezetimibe reduces sympa- thetic activity despite similar reductions in cholesterol levels," Journal of the American Society of Hypertension, vol. 8, no. 10, pp. 715-723, 2014.

[17] G. Mundy, R. Garrett, S. Harris et al., "Stimulation of bone formation in vitro and in rodents by statins," Science, vol. 286, no. 5446, pp. 1946-1949, 1999.

[18] M. Takenaka, K. Hirade, K. Tanabe et al., "Simvastatin stimulates VEGF release via p44/p42 MAP kinase in vascular smooth muscle cells," Biochemical and Biophysical Research Communications, vol. 301, no. 1, pp. 198-203, 2003.

[19] S. N. Raafat, R. M. Amin, M. M. Elmazar, M. M. Khattab, and A. S. El-Khatib, "The sole and combined effect of simvastatin and platelet rich fibrin as a filling material in induced bone defect in tibia of albino rats," Bone, vol. 117, pp. 60-69, 2018.

[20] J. Zhou, X. Gao, S. Huang et al., "Simvastatin improves the jaw bone microstructural defect induced by high cholesterol diet in rats by regulating autophagic flux," BioMed Research International, vol. 2018, Article ID 4147932, 9 pages, 2018.

[21] M. Yamashita, F. Otsuka, T. Mukai et al., "Simvastatin antagonizes tumor necrosis factor-alpha inhibition of bone morphogenetic proteins-2-induced osteoblast differentiation by regulating Smad signaling and Ras/Rho-mitogen-activated protein kinase pathway," The Journal of Endocrinology, vol. 196, no. 3, pp. 601-613, 2008.

[22] A. Neve, A. Corrado, and F. P. Cantatore, "Osteoblast physiology in normal and pathological conditions," Cell and Tissue Research, vol. 343, no. 2, pp. 289-302, 2011.

[23] A. N. Tsartsalis, C. Dokos, G. D. Kaiafa et al., "Statins, bone formation and osteoporosis: hope or hype?," Hormones (Athens, Greece), vol. 11, no. 2, pp. 126-139, 2012.

[24] M. Niu, X. Feng, and L. Zhou, "The role of the ERK1/2 pathway in simvastatin-loaded nanomicelles and simvastatin in regulating the osteogenic effect in MG63 cells," International Journal of Nanomedicine, vol. Volume 13, pp. 8165-8178, 2018.

[25] P. Y. Chen, J. S. Sun, Y. H. Tsuang, M. H. Chen, P. W. Weng, and F. H. Lin, "Simvastatin promotes osteoblast viability and differentiation via Ras/Smad/Erk/BMP-2 signaling pathway," Nutrition Research, vol. 30, no. 3, pp. 191-199, 2010.

[26] N. Ghosh-Choudhury, C. C. Mandal, and G. G. Choudhury, "Statin-induced Ras activation integrates the phosphatidylinositol 3-kinase signal to Akt and MAPK for bone morphogenetic protein-2 expression in osteoblast differentiation," The Journal of Biological Chemistry, vol. 282, no. 7, pp. 4983-4993, 2007.

[27] P. Gazzerro, M. C. Proto, G. Gangemi et al., "Pharmacological actions of statins: a critical appraisal in the management of cancer," Pharmacological Reviews, vol. 64, no. 1, pp. 102-146, 2011.

[28] A. Moshiri, A. M. Sharifi, and A. Oryan, "Role of simvastatin on fracture healing and osteoporosis: a systematic review on in vivo investigations," Clinical and Experimental Pharmacology \& Physiology, vol. 43, no. 7, pp. 659-684, 2016.

[29] M. Schachter, "Chemical, pharmacokinetic and pharmacodynamic properties of statins: an update," Fundamental \& Clinical Pharmacology, vol. 19, no. 1, pp. 117-125, 2005.

[30] G. G. Walmsley, A. McArdle, R. Tevlin et al., "Nanotechnology in bone tissue engineering," Nanomedicine: Nanotechnology, Biology and Medicine, vol. 11, no. 5, pp. 1253-1263, 2015.

[31] G. Gaucher, R. H. Marchessault, and J. C. Leroux, "Polyesterbased micelles and nanoparticles for the parenteral delivery 
of taxanes," Journal of Controlled Release, vol. 143, no. 1, pp. 212, 2010.

[32] V. P. Torchilin, "Structure and design of polymeric surfactantbased drug delivery systems," Journal of Controlled Release, vol. 73, no. 2-3, pp. 137-172, 2001.

[33] H. Danafar, A. Sharafi, M. H. Kheiri, and S. Andalib, "Sulforaphane delivery using $\mathrm{mPEG}-\mathrm{PCL}$ co-polymer nanoparticles to breast cancer cells," Pharmaceutical Development and Technology, vol. 22, no. 5, pp. 642-651, 2017.

[34] Z. Karami, S. Sadighian, K. Rostamizadeh, M. Parsa, and S. Rezaee, "Naproxen conjugated mPEG-PCL micelles for dual triggered drug delivery," Materials Science \& Engineering. C, Materials for Biological Applications, vol. 61, pp. 665-673, 2016.

[35] D. T. Pham, A. Chokamonsirikun, V. Phattaravorakarn, and W. Tiyaboonchai, "Polymeric micelles for pulmonary drug delivery: a comprehensive review," Journal of Materials Science, vol. 56, no. 3, pp. 2016-2036, 2021.

[36] W. L. Yu, T. W. Sun, C. Qi et al., "Enhanced osteogenesis and angiogenesis by mesoporous hydroxyapatite microspheresderived simvastatin sustained release system for superior bone regeneration," Scientific Reports, vol. 7, no. 1, article 44129, 2017.

[37] A. Oryan, A. Kamali, and A. Moshiri, "Potential mechanisms and applications of statins on osteogenesis: current modalities, conflicts and future directions," Journal of Controlled Release, vol. 215, pp. 12-24, 2015.

[38] A. S. Chauhan, A. Maria, and A. Managutti, "Efficacy of simvastatin in bone regeneration after surgical removal of mandibular third molars: a clinical pilot study," Journal of Maxillofacial and Oral Surgery, vol. 14, no. 3, pp. 578-585, 2015.

[39] A. Gouda, E. Helal, S. Ali, S. Bakry, and S. Yassin, "Maxillary sinus lift using osteoinductive simvastatin combined with $\beta$ TCP versus $\beta$-TCP - a comparative pilot study to evaluate simvastatin enhanced and accelerated bone formation," Acta Odontologica Scandinavica, vol. 76, no. 1, pp. 39-47, 2018.

[40] S. Gupta, M. del Fabbro, and J. Chang, "The impact of simvastatin intervention on the healing of bone, soft tissue, and TMJ cartilage in dentistry: a systematic review and meta-analysis," International Journal of Implant Dentistry, vol. 5, no. 1, p. 17, 2019.

[41] M. L. Ho, Y. C. Fu, Y. H. Wang, C. H. Chen, G. J. Wang, and G. J. Wang, "Combination of calcium sulfate and simvastatin-controlled release microspheres enhances bone repair in critical-sized rat calvarial bone defects," International Journal of Nanomedicine, vol. 10, p. 7231, 2015.

[42] X. Liu, X. Li, L. Zhou et al., "Effects of simvastatin-loaded polymeric micelles on human osteoblast-like MG-63 cells," Colloids and Surfaces. B, Biointerfaces, vol. 102, pp. 420-427, 2013.

[43] L. B. Ferreira, V. Bradaschia-Correa, M. M. Moreira, N. D. Marques, and V. E. Arana-Chavez, "Evaluation of bone repair of critical size defects treated with simvastatin-loaded poly(lactic-co-glycolic acid) microspheres in rat calvaria," Journal of Biomaterials Applications, vol. 29, no. 7, pp. 965-976, 2014.

[44] I. C. Tai, Y. C. Fu, C. K. Wang, J. K. Chang, and M. L. Ho, "Local delivery of controlled-release simvastatin/PLGA/HAp microspheres enhances bone repair," International Journal of Nanomedicine, vol. 8, p. 3895, 2013.

[45] M. Nyan, T. Miyahara, K. Noritake et al., "Molecular and tissue responses in the healing of rat calvarial defects after local application of simvastatin combined with alpha tricalcium phosphate," Journal of Biomedical Materials Research. Part B, Applied Biomaterials, vol. 9999B, 2009.

[46] S. Chen, J. Y. Yang, S. Y. Zhang, L. Feng, and J. Ren, "Effects of simvastatin gel on bone regeneration in alveolar defects in miniature pigs," Chinese Medical Journal, vol. 124, p. 3953, 2011.

[47] X. Yue, M. Niu, T. Zhang et al., "In vivo evaluation of a simvastatin-loaded nanostructured lipid carrier for bone tissue regeneration," Nanotechnology, vol. 27, no. 11, article 115708, 2016.

[48] Y. Lee, M. J. Schmid, D. B. Marx et al., "The effect of local simvastatin delivery strategies on mandibular bone formation in vivo," Biomaterials, vol. 29, no. 12, pp. 1940-1949, 2008.

[49] K. Zhang, Y. B. Fan, N. Dunne, and X. M. Li, "Effect of microporosity on scaffolds for bone tissue engineering," Regenerative Biomaterials, vol. 5, no. 2, pp. 115-124, 2018. 